Objective To develop a measure of maternal mood that provides novel answering and scoring formats, accounts for the spectrum of emotions and symptoms experienced by women in the perinatal period, and correlates with clinical diagnostic measures.

Methods A literature review was conducted to assess current understanding of diagnostic criteria for perinatal mental health conditions. Based on previous research with adjective checklists and women's free text responses to national maternity surveys in 2010 and 2014, scoping of the measure was undertaken. A list of 24 adjectives (12 positive items, 12 negative items) was determined for a prototype measure in which women could choose the adjectives to describe how they had been feeling in the last seven days. Cognitive interviews were conducted with 12 women who had recently given birth, and positive feedback endorsed the content, verified item selection and face validity of the scale.

Results The checklist was administered in a survey of maternal and child health to which 551 new mothers responded. Exploratory and Confirmatory factor analyses were conducted to explore underlying factor structure. Two models resulted: a two-factor solution (1. positive mood, 2. negative mood) and a four-factor solution: (1. positive mood, 2. negative mood, agitation; 3.anhedonia, low energy; 4. positive life orientation). Analyses were undertaken for validation and to explore associations with other screening measures to support its use.

Conclusion This novel method of reporting feelings and mood in an engaging format will facilitate research in the perinatal field and allow more opportunities for conversations about mood and mental health with health care professionals. As a tool that is psychometrically robust, time-efficient, and which may afford greater insight on the emotional state of the women cared for, the perinatal mood checklist is an effective addition to measures currently available.

\section{P79 VALIDATION OF TWO SECONDARY SOURCES OF FOOD ENVIRONMENT DATA AGAINST STREET AUDITS IN ENGLAND}

${ }^{1}$ E Wilkins*, ${ }^{2} \mathrm{M}$ Morris, ${ }^{1} \mathrm{D}$ Radley, ${ }^{1} \mathrm{C}$ Griffiths. ${ }^{1}$ Carnegie, Leeds Beckett University, Leeds, UK; ${ }^{2}$ Leeds Institute for Data Analytics, School of Medicine, University of Leeds, Leeds, UK

10.1136/jech-2017-SSMAbstracts. 180

Background Public Health England recently published a tool to help local authorities monitor the density of fast food outlets, with restrictions on access to these 'unhealthy' outlets being promoted in the fight against obesity. Secondary data containing the locations of food outlets present valuable resources to guide and evaluate these interventions. However, evidence appraising these data sources is limited. This study therefore seeks to validate two sources of secondary food environment data (SFED): Ordnance Survey Points of Interest (POI) data and food hygiene data from the Food Standards Agency (FSA), against street audits. These data sources are commonly used in research, with the former also being used in the Public Health England tool.

Methods Audits were conducted across 54 Lower Super Output Areas in England. All streets within each Lower Super Output Area were covered to identify the name and street address of all food outlets therein. Audit identified outlets were matched to outlets in the SFED to identify true positives (TP: outlets in both the SFED and the audits), false positives
(FP: outlets in the SFED only) and false negatives (FN: outlets in the audits only). Agreement was assessed using positive predictive values (PPV: TP/(TP +FP)) and sensitivities (TP/ $(\mathrm{TP}+\mathrm{FN}))$. Confidence intervals were calculated in Excel using the Agresti-Coull method.

Results Overall, the street audits identified 1188 food outlets, compared to 1102 and 1098 for the POI and FSA data respectively. Sensitivity and PPV were significantly higher for FSA data (sensitivity: 0.80, CI: 0.77-0.82; PPV: 0.86, CI: $0.84-0.88$ ) than for the POI data (sensitivity: 0.73 , CI: $0.71-$ 0.76; PPV: 0.79, CI: 0.77-0.81). Both datasets had 'good' agreement with street audits according to the Paquet classifications ('good' defined as PPV and sensitivities between 0.71 and 0.90 ).

Conclusion This study provides new evidence for the validity of SFED commonly used in research and emergently used by policymakers. Agreement between the SFED and street audits is sufficiently good to provide local authorities with confidence in using tools and research based on these SFED. Whilst FSA data has statistically significantly higher agreement with street audits than POI, the magnitude of the difference is relatively small. POI also has other advantages (e.g. more detailed outlet classifications and better spatial accuracy). Thus, POI is still a useful and recommended source of food environment data.

\section{P80 DOES SELF-RATED HEALTH MEASURE THE SAME CONCEPT ACROSS COUNTRIES? INSIGHTS FROM A COMPARISON OF OLDER ADULTS IN ENGLAND AND JAPAN}

${ }^{1}$ BD Williams*, ${ }^{1} \mathrm{~T}$ Chandola, ${ }^{2} \mathrm{~N}$ Cable. ${ }^{1}$ Cathie Marsh Institute for Social Research, University of Manchester, Manchester, UK; ${ }^{2}$ Institute of Epidemiology and Health Care, University College London, London, UK

\subsection{6/jech-2017-SSMAbstracts. 181}

Background Self-Rated Health (SRH) is predictive of morbidity and mortality, correlates well with objective measurements of physical function and is simple to use in multidisciplinary surveys. It could be a useful way of comparing health policies in different countries. However, it may not be comparable between countries which may wish to contrast health policies, for example Britain and Japan, because of linguistic, cultural or health differences. We aimed to test for differences in the association between SRH and physical function (grip strength), mental health (depression) and cardiovascular risk (smoking and BMI) between older adults in Japan and England.

Methods Data were used from the English Longitudinal Study of Ageing (ELSA; 2004, 2008 and 2012) and the Japanese Study of Ageing and Retirement (JSTAR; 2007, 2009 and 2011), giving $n=10,174$ ELSA participants and $n=4279$ JSTAR participants, all aged 50 and above. Multilevel binary logistic regression was used to test whether participants' country of residence was associated with odds of fair or poor $\mathrm{SRH}$ and whether the country of residence would moderate associations between SRH and grip strength, depression, smoking or BMI.

Results Japanese women (15.6\%) and men (14.1\%) were less likely to report fair/poor SRH than English women (23.6\%) and men (24.1\%). After adjusting for covariates these differences remained for men (Odds Ratio [OR] for Japanese men 0.64, 95\% CI 0.74-0.85) but not for women (OR for Japanese women 1.02, 95\% CI 0.47-1.34). Grip strength (OR's 
women 0.86-0.89; men 0.91-0.94), depression (OR's women 4.40-6.82; men 5.25-9.26), BMI (OR's women 1.11-1.16; men 1.10-1.15) and smoking status (OR's $\geq 20$ cigarettes per day women 3.07-7.22; men 2.13-4.54) were associated with fair/poor SRH. No interactions were found between country and grip strength (OR's women 0.95-1.03; men 0.99-1.05) or depression (OR's women 0.63-1.39; men 0.50-1.22) but were found for BMI (OR's women 0.89-0.98; men 0.87-0.97) and smoking (OR's $\geq 20$ cigarettes per day women 0.12-0.34; men $0.20-0.55)$. The interaction between country and BMI reduced when the analysis was restricted to those with a BMI less than 30 (OR's women 0.93-1.07; men 0.90-1.05).

Conclusion Our findings agree with previous research that SRH captures general physical and mental health similarly across countries. We may need more caution comparing SRH across countries when considering other aspects of health. We find that cardiovascular risk has different associations with $\mathrm{SRH}$ in England and Japan, possibly reflecting differences in cultural norms and different stages in their obesity and tobacco epidemics.

\section{P81 FORECASTING TRENDS IN DISABILITY IN ENGLAND AND WALES TO 2030: A MODELLING STUDY}

${ }^{1} \mathrm{M}$ Guzmán-Castillo*, ${ }^{2} \mathrm{~S}$ Ahmadi-Abhari, ${ }^{1,3} \mathrm{P}$ Bandosz, 'S Capewell, ${ }^{2} \mathrm{~A}$ Steptoe, ${ }^{2,4} \mathrm{~A}$ Singh-Manoux, ${ }^{2} \mathrm{M}$ Kivamaki, ${ }^{2} \mathrm{MJ}$ Shipley, ${ }^{2} \mathrm{EJ}$ Brunner, ${ }^{1} \mathrm{M}$ O'Flaherty. ${ }^{1}$ Department of Public Health and Policy, University of Liverpool, Liverpool, UK; ${ }^{2}$ Department Epidemiology and Public Health, University College London, London, UK; ${ }^{3}$ Department of Prevention and Medical Education, Medical University of Gdansk, Gdansk, Poland; ${ }^{4}$ nserm, U1018, Centre for Research in Epidemiology and Population Health, Villejuif, France

\subsection{6/jech-2017-SSMAbstracts.182}

Background Reliably estimating the future burden of disability in our ageing societies is crucial. However, previous forecasts failed to consider the potentially significant impact of trends in disease incidence, both up and down. Our aim is thus to forecast the future disability burden in England and Wales to 2030, while taking into account ongoing trends in CVD and dementia incidence.

Methods We developed and validated the IMPACT-Better Ageing Model. Using evidence-based age-sex- and year-specific transition probabilities, this probabilistic model tracks the England and Wales population aged 35-100 years through ten health states (notably CVD, cognitive impairment, disability, dementia and death). We projected continuing declines in dementia incidence, CVD incidence and mortality rates through to 2030 (based on ELSA analysis and consistent with cohorts elsewhere). We then estimated future disability prevalence, distinguishing four types of disability: CVD-related, dementia-related, CVD and dementia-related and Non-CVD/ Non-dementia disability.

Results England and Wales will continue its transition towards a much older population structure. Between 2015 and 2030, the number of people aged over 65 years will increase by approximately 33.2\% (95\% uncertainty interval 32.2\%-34.1\%) while the very old population (over 85) will increase by some $68.3 \%(63.2 \%-73.0 \%)$

The standardised prevalence of disability among adults aged over 65 years will stay constant at around $21.4 \%(21.2 \%-$ $21.6 \%)$. However, the number of people living with disability will rise by $40.1 \%$ from 2.3 million to 3.2 million.

In 2030, Non-CVD/Non-dementia related disability will account for approximately two-thirds of disabled people aged
65-84, (the absolute numbers increasing in approximately $52 \%$ from 2015). Dementia-related disability will also increase by some 51\% (44.4\%-58.8\%). Conversely, CVD-related disability will decline by approximately 27\% (22.2\%-30.0\%).

Among the very old aged over 85 years, $40 \%$ of the cases of disability in 2030 will be attributable to dementia, representing a 113.4\% (102.6\%-123.6\%) increase since 2015. Non-CVD/Non-dementia disability will rise even more, by approximately 159.2\% (148.4\%\%-169.1\% ). However, CVDrelated disability cases, will increase by just $2.8 \% \quad(-1.4 \%-$ 7.0\%).

Conclusion The number of older people with care needs will expand by approximately one third by 2030, mainly reflecting population ageing rather than higher prevalence rates of disability. This will pose a substantial societal challenge and increase the need for cost-effective long-term care in all its forms: institutional, home-based and informal.

Future research on the potential benefits of effective prevention strategies might therefore concentrate on the shared determinants of these non-communicable diseases.

\section{P82 AN ILLUSTRATION OF THE ANALYTICAL CHALLENGES DUE TO MATHEMATICAL COUPLING IN HEALTH GEOGRAPHY RESEARCH}

${ }^{1,2}{ }^{2}$ Berrie* ${ }^{3}$ PD Norman, ${ }^{1,2} \mathrm{PD}$ Baxter, ${ }^{1,2} \mathrm{MS}$ Gilthorpe. 'Leeds Institute for Data Analytics, University of Leeds, Leeds, UK; ${ }^{2}$ Division of Epidemiology and Biostatistics, University of Leeds, Leeds, UK; ${ }^{3}$ School of Geography, University of Leeds, Leeds, UK

\subsection{6/jech-2017-SSMAbstracts. 183}

Background It has been acknowledged that the use of ratio variables is problematic in regression analysis where ratios comprise common components and are present in both the dependent and independent constituents of the model, e.g. regression analyses of ratio variables with common population denominators. However, such ratio variables are ubiquitous in health research and their resultant mathematical coupling (MC) has not been investigated extensively in relation to studies in health geography, where common population denominators are frequently encountered. It is common, for instance, that area level measures for health outcomes are considered in relation to area levels of mortality and/or indicators of social deprivation, where the common denominator is the area population. Our study seeks to illustrate this issue and examines the implications of this form of $\mathrm{MC}$ from a causal inference perspective.

Methods We examine the impact of MC amongst ratio variables in regression analyses using simulated data based on the correlation structure and distribution of variables derived from the UK census. Specifically, we consider the proportion of limiting long term illness (LLTI) in relation to mortality rates and the Townsend Material Deprivation Score (constructed from percentages of the population of an area that are experiencing pre-defined properties). Simulations are constructed under the null hypothesis, i.e. there is no impact of an area measure of deprivation on the relationship between mortality and proportion of limiting long term illness. A causal framework is introduced utilising directed acyclic graphs (DAGs) to assess variable relationships and to suggest analytical strategies that mitigate any problems arising due to MC.

Results We show that artefactual relationships arise in the regression analyses of composite proportions due to MC: area measures of deprivation appear to influence the relationship 